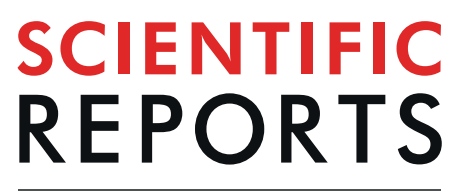

natureresearch

\title{
miRNA Expression Profiles and Potential as Biomarkers in Nontuberculous Mycobacterial Pulmonary Disease
}

\author{
Sun Ae Han $\mathbb{1}^{1,5}$, Byung Woo Jhun $\mathbb{1}^{1,5}$, Su-Young Kim ${ }^{1}$, Seong Mi Moon ${ }^{1}$, Bumhee Yang ${ }^{1}$, \\ O. Jung Kwon ${ }^{1}$, Charles L. Daley ${ }^{2,3}$, Sung Jae Shin $\mathbb{1}^{4 *}$ \& Won-Jung Koh ${ }^{1 *}$
}

Pulmonary disease (PD) due to nontuberculous mycobacteria (NTM) is increasing globally, but specific biomarkers for NTM-PD have not been established. As circulating miRNAs are promising biomarkers for various diseases, we investigated whether miRNAs have potential as NTM-PD biomarkers. Sera from 12 NTM-PD patients due to Mycobacterium avium, M. intracellulare, M. abscessus, or M. massiliense and three healthy controls were initially evaluated via small RNA sequencing. Multiple miRNAs showed significant differences in expression in patients compared to in healthy controls, with some expression differences unique to PD caused by a specific mycobacterial species. Notably, 14 miRNAs exhibited significant expression differences in PD associated with all four mycobacteria. Validation by quantitative reverse-transcription-PCR in an additional 40 patients with NTM-PD and 40 healthy controls confirmed that four differentially expressed miRNAs (hsa-miR-484, hsa-miR-584-5p, hsa-miR625-3p, and hsa-miR-4732-5p) showed significantly higher serum expressions in NTM-PD patients than in controls. Receiver operating characteristic curve analysis of these four miRNAs supported the discriminative potential for NTM-PD and their combination provided an improved diagnostic value for NTM-PD. Furthermore, bioinformatics analysis revealed their 125 target genes, which were mostly associated with immune responses. Collectively, this study identified four miRNAs as potential biomarkers for NTM-PD and provided insight into NTM-PD pathophysiology.

Nontuberculous mycobacteria (NTM) are mycobacteria other than the Mycobacterium tuberculosis complex and Mycobacterium leprae. More than 180 officially recognized NTM species have been identified, with the most frequent human pathogens associated with pulmonary disease (PD) due to NTM belonging to the Mycobacterium avium complex (MAC), followed by M. abscessus (MAB) ${ }^{1,2}$. MAC primarily comprises M. avium and M. intracellulare, and MAB primarily comprises M. abscessus subspecies abscessus (hereafter referred to as M. abscessus) and M. abscessus subspecies massiliense (hereafter referred to as M. massiliense) $)^{1,2}$. Despite the global increase in the burden of NTM-PD in recent decades, there is limited information regarding specific biomarkers for distinguishing patients with NTM-PD from healthy individuals or studies of the disease pathophysiology $\mathrm{y}^{3,4}$.

MicroRNAs (miRNAs) are small, 18-25-nucleotide, endogenous, stable, highly conserved noncoding RNAs with important functions in post-transcriptional gene regulation under both physiological and pathological conditions $^{5,6}$. Numerous studies have reported the aberrant expression of several miRNAs in various conditions, including cancer and infectious diseases. miRNAs reportedly play an important regulatory role in the pathogenesis or progression of infectious diseases 7,8 and serve as potential modulators of innate and adaptive immune responses $^{9,10}$. Serum miRNAs are not readily degraded by enzymes, unaffected by changes in temperature and time, and resistant to acids and alkalis ${ }^{11,12}$. Furthermore, serum miRNAs are stable, and evaluating serum miRNA profiles is a feasible diagnostic procedure in clinical laboratories. Because of their high diagnostic potential,

${ }^{1}$ Division of Pulmonary and Critical Care Medicine, Department of Medicine, Samsung Medical Center, Sungkyunkwan University School of Medicine, Seoul, South Korea. ${ }^{2}$ Division of Mycobacterial and Respiratory Infections, Department of Medicine, National Jewish Health, Denver, CO, USA. ${ }^{3}$ Department of Medicine, University of Colorado, Aurora, Colorado, USA. ${ }^{4}$ Department of Microbiology, Institute for Immunology and Immunological Disease, Brain Korea 21 PLUS Project for Medical Science, Yonsei University College of Medicine, Seoul, South Korea. ${ }^{5}$ These authors contributed equally: Sun Ae Han and Byung Woo Jhun. *email: sjshin@yuhs.ac; wjkoh@skku.edu 


\begin{tabular}{|l|l|l|}
\hline Characteristics & NTM-PD $(\mathbf{n}=\mathbf{5 2})$ & Healthy Controls $(\mathbf{n}=\mathbf{4 3})$ \\
\hline Age (years) & $56(50-62)$ & $48(40-56)$ \\
\hline Female sex & $45(87)$ & $29(67)$ \\
\hline Body mass index $\left(\mathrm{kg} / \mathrm{m}^{2}\right)$ & $20.8(19.0-22.5)$ & NA \\
\hline Never smoker & $46(89)$ & INA \\
\hline Etiologic organism & & INA \\
\hline M. avium & $13(25)$ & - \\
\hline M. intracellulare & $14(27)$ & - \\
\hline M. abscessus & $13(25)$ & - \\
\hline M. massiliense & $12(23)$ & - \\
\hline Underlying disease & \multicolumn{1}{|l|}{} \\
\hline Bronchiectasis & $48(92)$ & - \\
\hline Previously treated tuberculosis & $17(33)$ & - \\
\hline Previous thoracic surgery & $1(2)$ & - \\
\hline Chronic heart disease & $1(2)$ & - \\
\hline Chronic liver disease & $1(2)$ & - \\
\hline Laboratory data & & INA \\
\hline White blood cell $(/ \mu \mathrm{L})$ & $6040(4780-7813)$ & INA \\
\hline Lymphocyte $(\%)$ & $29.9(19.5-34.6)$ & INA \\
\hline Erythrocyte sedimentation rate $(\mathrm{mm} / \mathrm{hr})$ & $36(18-52)$ & INA \\
\hline Albumin $(\mathrm{g} / \mathrm{dl})$ & $4.5(4.3-4.6)$ & INA \\
\hline Computed tomography finding & & - \\
\hline Nodular bronchiectatic form & $48(92)$ & - \\
\hline Fibrocavitary form & $4(8)$ & - \\
\hline Positive acid fast stain & $31(60)$ & INA \\
\hline Forced expiratory volume in 1 second $(\%)$ & $79(72-93)$ & INA \\
\hline
\end{tabular}

Table 1. Demographic and clinical characteristics of study participants. Data are presented as no. (\%) or median (interquartile range). NTM-PD, nontuberculous mycobacterial pulmonary disease; INA, information not available.

serum miRNAs have been evaluated as biomarkers in several pathological conditions including TB, with some studies achieving $82 \%$ to $100 \%$ accuracy in diagnosing TB by evaluating miRNA ${ }^{13-18}$. Additionally, several studies have reported differences in miRNA levels among patients with active TB, latent TB, and healthy controls ${ }^{18,19}$. Recently, the roles of miRNAs in mycobacterial infections, particularly in tuberculosis (TB), has received increasing attention ${ }^{13,20-23}$ Such studies have reported that specific miRNAs are differentially expressed in macrophages or patients infected with $M$. tuberculosis complex and that several of these miRNAs interact with their cognate target genes. Additionally, studies have demonstrated that miRNAs are involved in the progression of TB and that serum miRNA profiles can be useful as potential diagnostic or therapeutic biomarkers in patients with $\mathrm{TB}^{13,20-23}$. The serum levels of miR-144 and miR-155, which were differentially expressed in patients with pulmonary TB compared to in healthy controls, have been reported to be diagnostic markers for $\mathrm{TB}^{24,25}$.

As the burden of NTM-PD is increasing worldwide, studies reporting on the expression of miRNA in NTM-PD have also increased. For example, overexpression of let-7e, miR-29a, and miR-886-5p inhibited $M$. avium-induced apoptosis in human monocyte-derived macrophages by modulating caspases 3 and $7^{26}$. Serum miR-346, expressed in $M$. avium-infected macrophages secreted into the bloodstream, was quantitatively controlled by bacterial load and identified as a potential biomarker of MAC-PD ${ }^{27}$. Although circulating miRNAs were recently recognized as promising disease biomarkers in infectious diseases, few studies have examined the role of miRNA in NTM-PD. Therefore, we evaluated serum miRNAs that are differentially expressed in NTM-PD patients compared to in healthy controls by RNA sequencing and explored the potency of serum miRNA expression profiles as discriminating markers for NTM-PD.

\section{Results}

Patients. In total, 95 participants were recruited in this study, including 52 patients with NTM-PD and 43 healthy volunteers (Table 1). The median age of the patients was 56 years, and 45 (87\%) were female. The median body mass index of the 52 patients was $20.8 \mathrm{~kg} / \mathrm{m}^{2}$, and $46(89 \%)$ were never smokers. Among the patients with NTM-PD, the most common underlying respiratory disease was bronchiectasis $(n=48,92 \%)$, followed by previous treated TB $(\mathrm{n}=17,33 \%)$. The median value of white blood cell count and erythrocyte sedimentation rate was $6040 / \mu \mathrm{L}$ and $36 \mathrm{~mm} / \mathrm{hr}$, respectively. Out of the 52 patients with NTM-PD, 48 (92\%) had nodular bronchiectatic form, and 4 (8\%) had the fibrocavitary form (Supplementary Fig. S1). The median forced expiratory volume in 1 second (\%) on pulmonary function test at diagnosis was 79\%. In the 43 healthy controls, the median age was 48 years, and $29(67 \%)$ patients were female.

Illumina-based small RNA sequencing of serum miRNAs. Serum levels of miRNA from 12 patients with NTM-PD, including patients infected with M. avium, M. intracellulare, M. abscessus, or M. massiliense ( $\mathrm{n}=3$ 


\begin{tabular}{|l|l|l|}
\hline & NTM-PD & Healthy controls \\
\hline Total reads & $11,217,363$ & $18,556,427$ \\
\hline Precursor miRNA reads & 3,445 & 8,444 \\
\hline Mature miRNA reads & $7,250,677$ & $5,265,841$ \\
\hline No. of known miRNAs & 467 & 407 \\
\hline No. of novel miRNAs & 262 & 193 \\
\hline Distribution of the genome-mapped sequence reads \\
\hline miRNA & $3,517,419(39.7)$ & $3,083,932(44.5)$ \\
\hline lincRNA & $2,397,431(27.0)$ & $1,655,179(23.9)$ \\
\hline rRNA & $10,857(0.1)$ & $6,326(0.1)$ \\
\hline snoRNA & $8,987(0.1)$ & $5,626(0.1)$ \\
\hline snRNA & $25,265(0.3)$ & $20,821(0.3)$ \\
\hline others & $2,924,390(32.8)$ & $2,164,554(31.2)$ \\
\hline Distribution of genomic repetitive sequences & \multicolumn{2}{|l|}{} \\
\hline LINE (sense) & $280,607(27.0)$ & $237,082(26.4)$ \\
\hline LINE (anti sense) & $155,079(14.9)$ & $136,196(15.2)$ \\
\hline LTR (sense) & $148,237(14.3)$ & $134,467(15.0)$ \\
\hline LTR (anti sense) & $105,699(10.2)$ & $108,196(12.1)$ \\
\hline SINE (sense) & $124,916(12.0)$ & $101,246(11.3)$ \\
\hline SINE (anti sense) & $118,899(11.4)$ & $87,079(9.7)$ \\
\hline Retroposon (sense) & $39675(3.8)$ & $39720(4.4)$ \\
\hline Retroposon (anti sense) & $64934(6.3)$ & $52578(5.9)$ \\
\hline others & $766(0.1)$ & $696(0.1)$ \\
\hline & & \\
\hline
\end{tabular}

Table 2. The number of miRNAs in serum samples from patients with NTM-PD and healthy controls by small RNA sequencing analysis. Data are presented as no. (\%). NTM-PD, nontuberculous mycobacterial pulmonary disease; miRNA, microRNA; lincRNA, long intergenic noncoding RNA; rRNA, ribosomal RNA; snoRNA, small nucleolar RNA; snRNA, small nuclear RNA; LINE, long interspersed nuclear element; LTR, long terminal repeat; SINE short interspersed nuclear element.

each), and three healthy individuals were examined by Illumina small RNA sequencing in the discovery phase. Averages of 11,217,363 and 18,556,427 reads of RNAs ranging from 18 to 30 nucleotides were obtained from pooled serum samples of patients with NTM-PD and healthy controls, respectively. The length distribution of clean sequences in the reference genome was determined. Length distribution analysis of serum pools from both patients with NTM-PD and healthy controls revealed that most reads were in the range of $18-24 \mathrm{nt}$, which is consistent with the common length of miRNAs ${ }^{28,29}$. The three pools of serum samples contained small RNAs of various lengths (Supplementary Fig. S2). Thereafter, bioinformatics analysis was performed to investigate the small RNA species and sequencing frequencies.

In serum derived from patients with NTM-PD and healthy controls, multiple and heterogeneous small RNA species, including miRNAs, long intergenic noncoding RNA, ribosomal, small nucleolar, and small nuclear RNA were identified (Table 2). In serum samples of patients with NTM-PD and healthy controls, miRNAs accounted for $39.7 \%$ and $44.5 \%$, respectively, of the total amount of small RNAs (sequencing reads), and there was no significant difference between the two groups. We analyzed all clean reads using mirDeep software to identify known and novel miRNAs. In total, 467 and 407 known miRNAs were identified among patients with NTM-PD and healthy controls, respectively (Table 2). Novel miRNA precursors were predicted, and an average of 262 miRNAs in patients with NTM-PD and 193 miRNAs in healthy controls were identified (Table 2).

In addition, differentially expressed miRNAs were observed in the serum miRNA profiles of patients with NTM-PD relative to in healthy controls (Fig. 1). We identified 148 differentially expressed miRNAs based on two-fold changes and p-values less than 0.05 . Of these 148 miRNAs, 70 miRNAs showed significant differences in expression in patients with M. avium-PD, 56 miRNAs in patients with M. intracellulare-PD, 46 miRNAs in patients with M. abscessus-PD, and 32 miRNAs in patients with $M$. massiliense-PD compared to in healthy controls (Supplementary Tables S1-4, Fig. 2a-d). However, only 14 of these miRNAs were differentially expressed in all four patient groups, i.e., in PD due to any of the four NTM organisms (M. avium, M. intracellulare, M. abscessus, and M. massiliense) (Fig. 2e,f).

Differential expression of miRNAs via qRT-PCR analysis. As the 14 miRNAs with differential expression in all four patient groups were considered as potential biomarkers, their expression was verified by qRT-PCR analysis. In the selection phase, the 14 candidate miRNAs were measured in a separate set of individual serum samples from 12 patients with NTM-PD and three healthy controls, i.e., the number of the same subjects used for small RNA sequencing. miRNAs with a mean fold-change (NTM-PD/healthy controls) of $>2$ and $p$-values $<$ 0.05 were selected for further analysis. The analysis indicated that five miRNAs (hsa-miR-423-5p, hsa-miR-484, hsa-miR-584-5p, hsa-miR-625-3p, and hsa-miR-4732-5p) of the 14 candidates showed significant differences in expression between patients with NTM-PD and healthy controls (Fig. 3). 


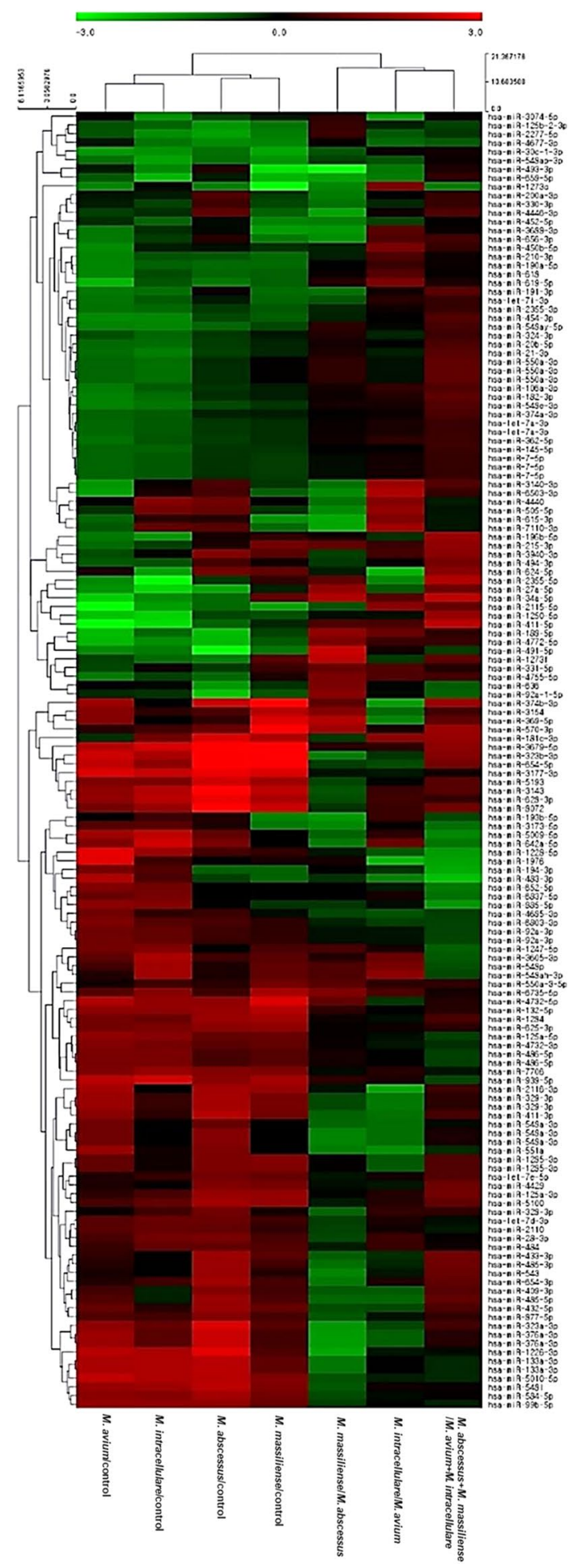

Figure 1. Hierarchical clustering of differentially expressed microRNAs in sera of patients with NTM-PD and healthy controls. A total of 148 miRNAs were differentially expressed with separate clustering of NTM-PD patients and healthy controls. Each row represents the expression of a given gene in each sample, and each column represents the expression of each gene in a given sample. Rows and columns are hierarchically clustered. Red and Green colors represent up- and downregulation of miRNAs in NTM-PD patients relative to levels in healthy controls.

qRT-PCR-based validation was performed to quantify differences in the expression levels of the five miRNAs in samples from 40 healthy controls and 40 patients with NTM-PD (including NTM-PD due to M. avium, M. intracellulare, $M$. abscessus, and M. massiliense; $\mathrm{n}=10$ each). Apart from hsa-miR-423-5p $(p=0.116)$, four of the five miRNAs, i.e., hsa-miR-484 $(p=0.015)$, hsa-miR-584-5p $(p=0.001)$, hsa-miR-625-3p $(p=0.002)$, and 
(a)

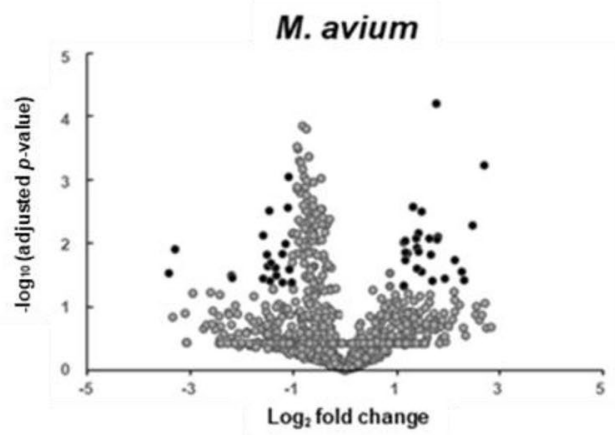

(c)

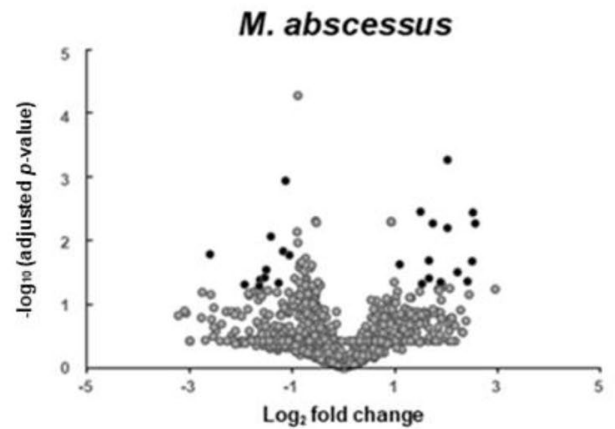

(e)

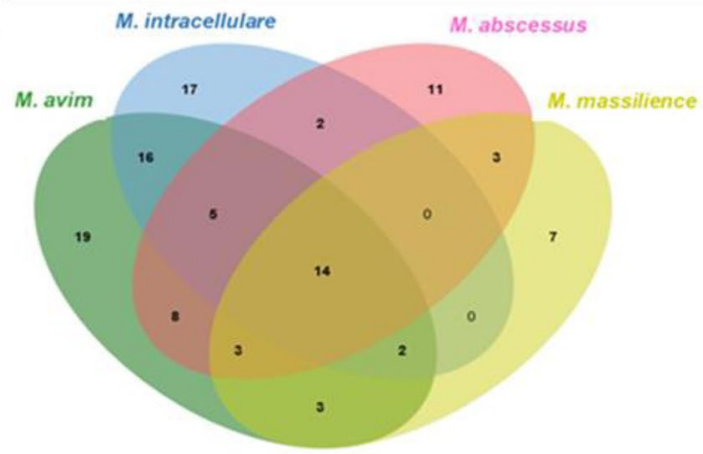

(b)

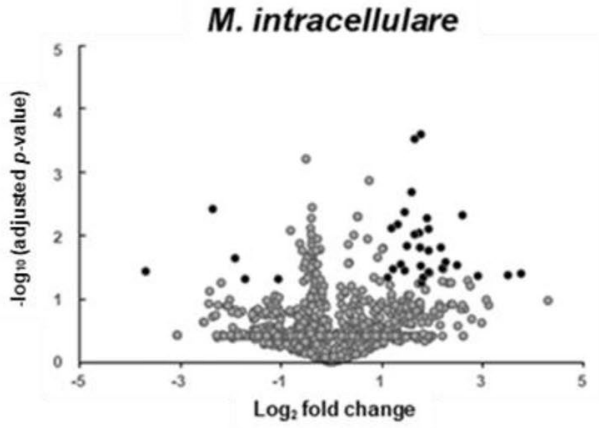

(d)

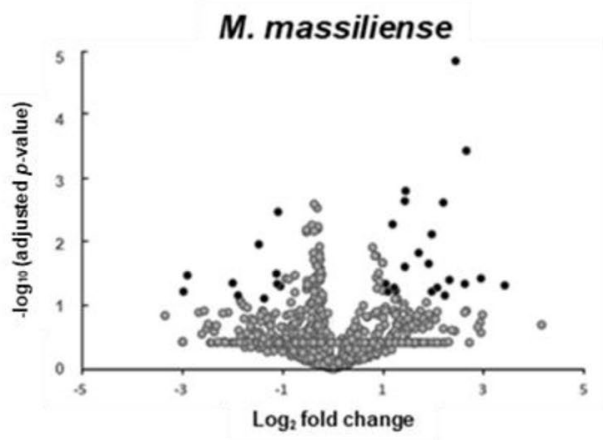

(f)

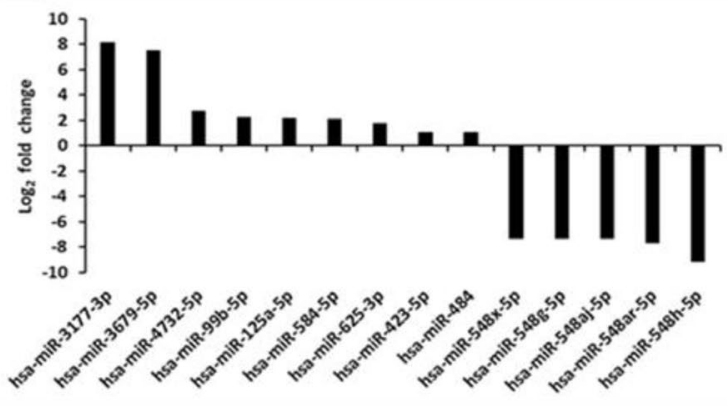

Figure 2. Volcano plots and Venn diagram of differentially expressed miRNAs in NTM-PD. (a-d) Volcano plots representing $\log 2$ fold change as a function of $-\log _{10}$ (the adjusted p-value) for miRNA expression in NTM-PD versus healthy controls. miRNAs found to be differentially expressed between the NTM-PD patients and controls are shown in black and unchanged miRNAs are shown in grey. (a) Mycobacterium avium patients, (b) M. intracellulare patients, (c) M. abscessus patients, and (d) M. massiliense patients. (e) Venn diagram showing miRNAs significantly expressed in NTM-PD patients versus healthy controls. (f) Histogram of the 14 miRNAs that were differentially expressed in NTM-PD from all four NTM species. Expression is shown as fold change over levels in healthy controls.

has-miR-4732-5p $(p=0.014)$, showed significantly higher serum expression levels in patients with NTM-PD than in healthy controls (Fig. 3).

Receiver operating characteristic (ROC) curve analysis of four miRNAs. Using validation data obtained from the 40 patients with NTM-PD and 40 healthy controls, ROC curve analysis was performed to determine how well the selected serum miRNAs could distinguish between the two groups. miR-423-5p $(p=0.116)$ were removed based on $p$-values $>0.05$. ROC curves were constructed to compare the relative expression levels of the four miRNAs between the two groups (Table 3, Fig. 4). The areas under the curves (AUC) of has-miR-4732-5p for distinguishing NTM-PD from healthy controls was $0.892(p<0.0001,95 \% \mathrm{CI}=0.800-0.952)$, which was comparable to that of hsa-miR-484, $0.741(p<0.0001,95 \% \mathrm{CI}=0.631-0.833)$, hsa-miR-584-5p, $0.712(p<0.0001$, $95 \% \mathrm{CI}=0.597-0.810)$, and hsa-miR-625-3p, $0.773(p<0.0001,95 \% \mathrm{CI}=0.665-0.860)$ (Fig. 4a-d). Multivariate 
a

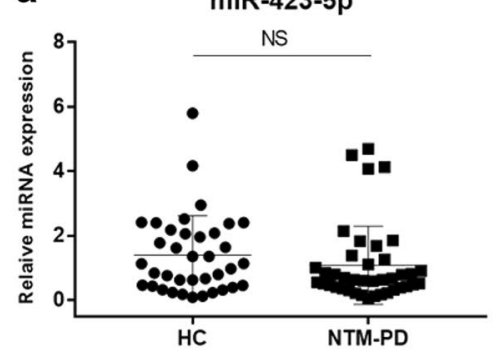

C

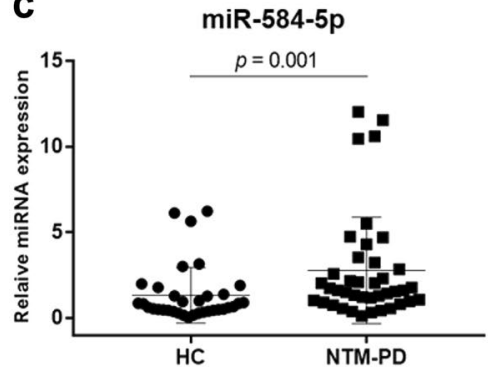

e $\quad \operatorname{miR}-4732-5 p$

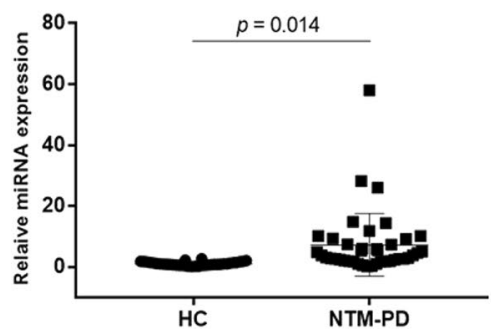

b

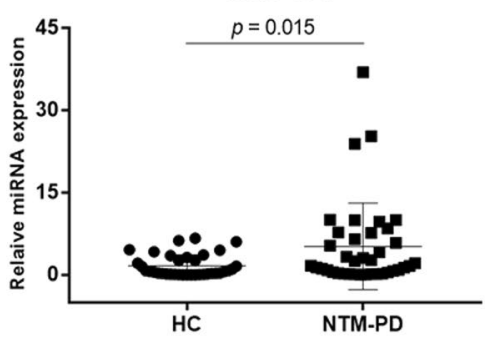

d

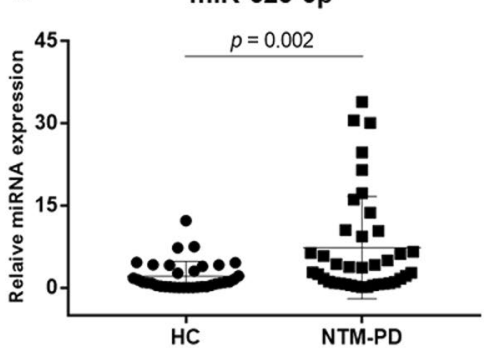

Figure 3. Validation of selected miRNA expression levels by qRT-PCR in NTM-PD patients. Serum levels of the five miRNAs were measured in 40 NTM-PD patients and in 40 healthy controls. Expression levels of the miRNAs are normalized to miR-16. (a) has-miR-423-5p, (b) has-miR-484, (c) has-miR-584-5p, (d) has-miR625-3p, and (e) has-miR-4732-5p. qRT-PCR, quantitative real-time PCR; HC, healthy controls; NTM-PD, nontuberculous mycobacterial pulmonary disease; NS, not significant.

logistic regression analysis in ROC curve was used to identify the optimal combination of four miRNAs to discriminate NTM-PD. The integration of has-miR-4732-5p with hsa-miR-484 and hsa-miR-625-3p (AUC 0.998; sensitivity 95\%; and specificity 100\%) resulted in an improved diagnostic value for NTM-PD (Fig. 4e).

Construction of miRNA-gene networks. To further investigate the putative functions of the candidate miRNAs, the TargetScan database (http://targetscan.org) was used to analyze the target mRNAs, and the Database for Annotation, Visualization, and Integrated Discovery (http://david.abcc.ncifcrf.gov/) was used to identify the biochemical pathways associated with the conserved targets. Figure 5 shows the miRNA-gene network for hsa-miR-484, hsa-miR-584-5p, hsa-miR-625-3p, and hsa-miR-4732-5p. These four miRNAs were associated with $4231,2268,2475$, and 2242 target mRNAs in the TargetScan database, respectively. Among these genes, 125 genes, including nine genes related to TB, were predicted to be targeted by the four miRNAs in common (Fig. $5 \mathrm{a}-\mathrm{c}$ ). For example, hsa-miR-484, hsa-miR-584-5p, hsa-miR-625-3p, and hsa-miR-4732-5p all targeted the nuclear factor of activated T cells 5 (NFAT5) and Toll-like receptor 4 (TLR4). Hsa-miR-484, hsa-miR-584-5p, and hsa-miR4732-5p targeted interleukin 6 (IL-6), and hsa-miR-484 and hsa-miR-625-3p targeted interleukin-17 (IL-17). Has-miR-484 targeted CD40 and the CD40 ligand. Hsa-miR-625-3p targeted CD28, a coreceptor that mediates $\mathrm{T}$ cell activation.

Go analysis and KEGG pathway analysis of target genes. Gene ontology (GO) and the Kyoto Encyclopedia of Genes and Genomes (KEGG) pathway analysis were applied to further classify the identified genes into the functional groups. GO enrichment analyses were performed to classify 125 target genes based on biological processes, molecular function, and cellular component (Fig. 6a). In the case of biological processes, the genes were classified into forty-six categories, of which the most significant term was regulation of transcription and DNA-template (GO: 0006355). Based on cellular components, the genes were classified into ten categories, of which the most significant term was mitochondrial lumen (GO: 0005759). Based on molecular function, the 


\begin{tabular}{|l|l|l|l|}
\hline miRNA & AUC $(95 \%$ CI $)$ & Sensitivity,\% & Specificity, \% \\
\hline has-miR-484 & $0.741(0.631-0.833)$ & 80.0 & 70.0 \\
\hline has-miR-584-5p & $0.712(0.597-0.810)$ & 75.0 & 66.7 \\
\hline has-miR-625-3p & $0.773(0.665-0.860)$ & 60.0 & 89.7 \\
\hline has-miR-4732-5p & $0.892(0.800-0.952)$ & 80.0 & 91.7 \\
\hline $\begin{array}{l}\text { has-miR-4732-5p+ has-miR-484+ has-miR-584-5p+ } \\
\text { has-miR-625-3p }\end{array}$ & $0.894(0.802-0.953)$ & 95.0 & 71.4 \\
\hline has-miR-4732-5p+ has-miR-484+ has-miR-584-5p & $0.905(0.813-0.962)$ & 82.5 & 90.6 \\
\hline has-miR-4732-5p+ has-miR-484+ has-miR-625-3p & $0.998(0.949-1.000)$ & 95.0 & 100.0 \\
\hline has-miR-4732-5p+ has-miR-584-5p+has-miR-625-3p & $0.995(0.941-1.000)$ & 97.5 & 96.9 \\
\hline has-miR-4732-5p+ has-miR-484 & $0.908(0.819-0.962)$ & 87.5 & 86.1 \\
\hline has-miR-4732-5p+ has-miR-584-5p & $0.888(0.791-0.950)$ & 80.0 & 90.6 \\
\hline has-miR-4732-5p+ has-miR-625-3p & $0.996(0.945-1.000)$ & 97.5 & 97.2 \\
\hline has-miR-484+ has-miR-584-5p & $0.776(0.666-0.863)$ & 80.0 & 69.4 \\
\hline has-miR-484+ has-miR-625-3p & $0.810(0.706-0.890)$ & 65.0 & 87.2 \\
\hline has-miR-584-5p+ has-miR-625-3p & $0.867(0.769-0.935)$ & 92.5 & 74.3 \\
\hline
\end{tabular}

Table 3. Comparison of the AUC, the sensitivity, and specificity for predicting NTM-PD. AUC, area under the curve; NTM-PD, nontuberculous mycobacterial pulmonary disease; miRNA, microRNA.

genes were classified into eleven categories, of which the most significant term was transition metal ion binding (GO: 0046914).

KEGG pathway analysis indicated that 125 putative target genes were mainly involved in central carbon metabolism in cancer, proteoglycans in cancer, inositol phosphate metabolism, endocytosis, adherent junction, Wnt signaling pathway, lysine degradation, glycosaminoglycan biosynthesis, cell cycle, ECM receptor interaction, hippo signaling pathway, and fatty acid biosynthesis (Fig. 6b).

\section{Discussion}

This study was conducted to compare the expression profiles of circulating serum miRNA from NTM-PD and healthy controls. In the discovery phase, 70 miRNAs in patients with M. avium-PD, 56 miRNAs in patients with $M$. intracellulare-PD, 46 miRNAs in patients with M. abscessus-PD, and 32 miRNAs in patients with $M$. massiliense-PD showed significant differences in expression relative to levels in healthy controls. Differential expression of 14 of these miRNAs was common to PD caused by any of the four organisms. In the validation phase, using qRT-PCR analysis of sera from additional patients and controls, we confirmed that four miRNAs (hsa-miR-484, hsa-miR-584-5p, hsa-miR-625-3p, and hsa-miR-4732-5p) of the 14 miRNAs were differentially expressed in patients. We further determined that 125 gene targets were shared by the candidate miRNAs and were associated with NTM-PD on TargetScan analysis. The present study is the first to demonstrate that hsa-miR-484, hsa-miR-584-5p, hsa-miR-625-3p, and hsa-miR-4732-5p are associated with NTM-PD and are potential biomarkers for NTM-PD. This study is valuable because multiple patients with NTM-PD and disease due to several NTM organisms were examined.

In the present study, four serum miRNAs (hsa-miR-484, hsa-miR-584-5p, hsa-miR-625-3p, and hsa-miR4732-5p) showed increased expression in patients with NTM-PD compared to in healthy controls. Interestingly, these four miRNAs were previously implicated in infection with Mycobacterium bovis bacillus Calmette-Guérin (BCG) and immune diseases. miR-484 is upregulated in exosomes of BCG-infected macrophages and targets mitochondrial cleavage protein 1 to modulate the intermediate metabolic pathway ${ }^{30}$. Various assays have revealed that miR-484 upregulation clearly influences cell migration and cellular proliferative capacity, and miR-484 is also tumorigenic, promoting the progression of non-small cell lung cancer ${ }^{31}$. miR-584 is reportedly upregulated in breast cancer cells, enhancing nuclear factor kappa B signaling, and inhibiting TGF- $\beta$ signaling ${ }^{32}$. TGF- $\beta$ has several inhibitory effects on T cells, B cells, macrophages, and other cells, and increased TGF- $\beta$ levels are associated with protection and/or recovery from autoimmune diseases ${ }^{33}$. Previous studies reported that miRNA-625-3p in the blood facilitated the detection of malignancy in benign lung tumors ${ }^{34}$, and miRNA-625-3p is reportedly upregulated in the urine of pulmonary TB patients and is an excellent diagnostic urinary biomarker ${ }^{35}$. miR$4732-3 p$ is associated with breast cancer and stomach cancer and is specifically expressed in exosomes released from macrophages after infection with $\mathrm{BCG}^{20,36}$. We hypothesized that differential expression of these miRNAs in patients with NTM-PD and healthy controls would result in differences of target mRNA expression; thus, additional experimental validation is further required.

In the present study, 125 genes were predicted to be targeted by the four miRNAs, including nine genes related to $M$. tuberculosis infection. Our results revealed that various genes associated with the innate immune response, cell proliferation, and apoptosis are potential targets of hsa-miR-484, hsa-miR-584-5p, hsa-miR-625-3p, and hsa-miR-4732-5p. Our results further showed that NFAT5 is a target gene for all four miRNAs; NFAT5 plays an important role in modulating HIV-1 replication by $M$. tuberculosis during co-infection, by directly interacting with the viral promoter ${ }^{37}$. Hsa-miR-484, hsa-miR-584-5p, and hsa-miR-4732-5p also targeted IL-6, a pleiotropic cytokine that plays a central role in host defense ${ }^{38}$. These results suggest that the four miRNAs play an important role in host-pathogen interactions. 
(a)

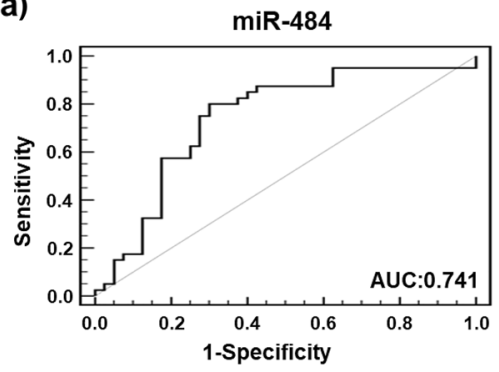

(c)

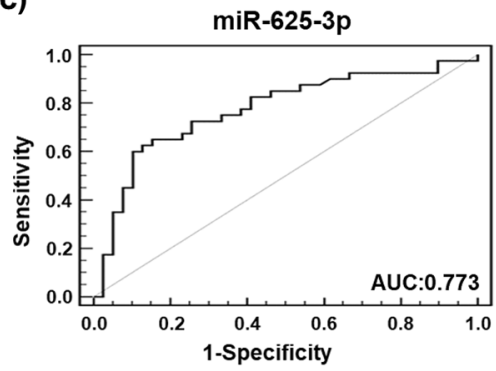

(b)

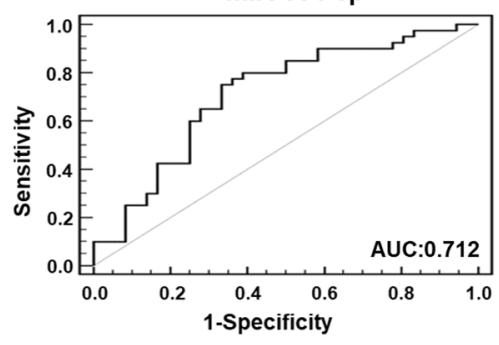

(d)

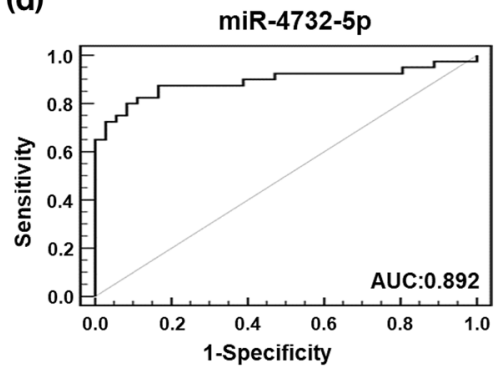

(e)

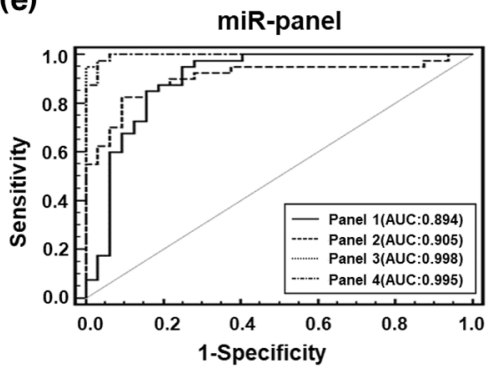

Figure 4. Receiver operating characteristic (ROC) curves of four selected miRNAs in NTM-PD patients. The ROC curves were used to show the discriminative ability of the selected four miRNAs with sensitivity (true-positive rate) and 1-specificity (false-positive rate). The $\mathrm{x}$-axis shows 1-specificity, and the y-axis shows sensitivity. Multivariate logistic regression analysis was performed, and ROC curves were generated to evaluate the ability of the chosen miRNAs to distinguish NTM-PD patients from controls. (a) has-miR-484, (b) has-miR584-5p, (c) has-miR-625-3p, (d) has-miR-4732-5p, and (e) has-miR-panel. AUC, area under the curve; Panel 1, has-miR-484+ has-miR-584-5p+ has-miR-625-3p+ has-miR-4732-5p; Panel 2, has-miR-484+ has-miR584-5p+ has-miR-4732-5p; Panel 3, has-miR-484+ has-miR-625-3p+ has-miR-4732-5p; Panel 4, has-miR584-5p+ has-miR-625-3p+ has-miR-4732-5p.

We performed comprehensive GO enrichment and KEGG pathway analysis of the target genes of the differentially expressed miRNAs. GO analysis indicated that these genes are involved in immune responses and oxidation-reduction processes and in the regulation of tumor necrosis factor- $\alpha$. KEGG analysis revealed the involvement of these genes in cell growth, migration, and proliferation and in pathways such as Hippo signaling, Wnt signaling, p53 signaling, and TGF- $\beta$ signaling. Thus, these miRNAs may influence NTM infection, as they affect immune cell development. In addition, most proteins encoded by the target genes are involved in mitogen-activated protein kinase signaling, Wnt signaling, and TGF- $\beta$ signaling in NTM-PD and may be useful in elucidating the pathogenesis of NTM-PD.

To further evaluate the diagnostic value of hsa-miR-484, hsa-miR-584-5p, hsa-miR-625-3p, and hsa-miR4732-5p, we performed a more detailed analysis using ROC curves. The present study found that the ROC curve of the combined four differentially expressed miRNAs showed an optimal discriminative ability with an AUC of 0.998 (95\% CI $=0.949-1.000)$, and diagnostic sensitivity and specificity of 95 and $100 \%$, respectively. Our data showed that the diagnostic value of these four miRNAs combination was higher than that of the individual miRNAs, indicating that the combinational signature of these four miRNA could serve as discriminating markers for NTM-PD.

There are several limitations to the current study. First, the number of samples in this study was small. Second, the present study did not include patient subjects with other lung diseases as controls for NTM-PD. To further validate the four miRNAs in discriminating NTM-PD from other lung diseases, appropriate PD control groups are required to confirm whether our findings are unique to NTM-PD. Finally, the combination sets of miRNAs target prediction were solely based on TargetScan database. Thus, the use of efficient genetic algorithms for variable selection in logistic regression model, rather than a linear model, may have advantages to select the clinically optimal markers among all extracted data sets, more accurately ${ }^{39}$.

In conclusion, to our best knowledge, this is the first study to determine the serum miRNA profiles in NTM-PD through deep sequencing. We identified 14 differentially expressed miRNAs in patients with NTM-PD compared to in healthy individuals, including nine upregulated miRNAs and five downregulated miRNAs. The present study verified the relative expression levels of hsa-miR-484, hsa-miR-584-5p, hsa-miR-625-3p, and hsa-miR-4732-5p, indicating their potential as biomarkers for NTM-PD. GO enrichment and KEGG analysis showed that the target genes of the four differentially expressed miRNAs were mainly involved in the immune response. These results indicate the presence of a serum-specific miRNA signature in patients with NTM-PD, which may modulate host antibacterial pathways in response to NTM infection and can be considered a potential molecular tool for diagnosing NTM-PD. 

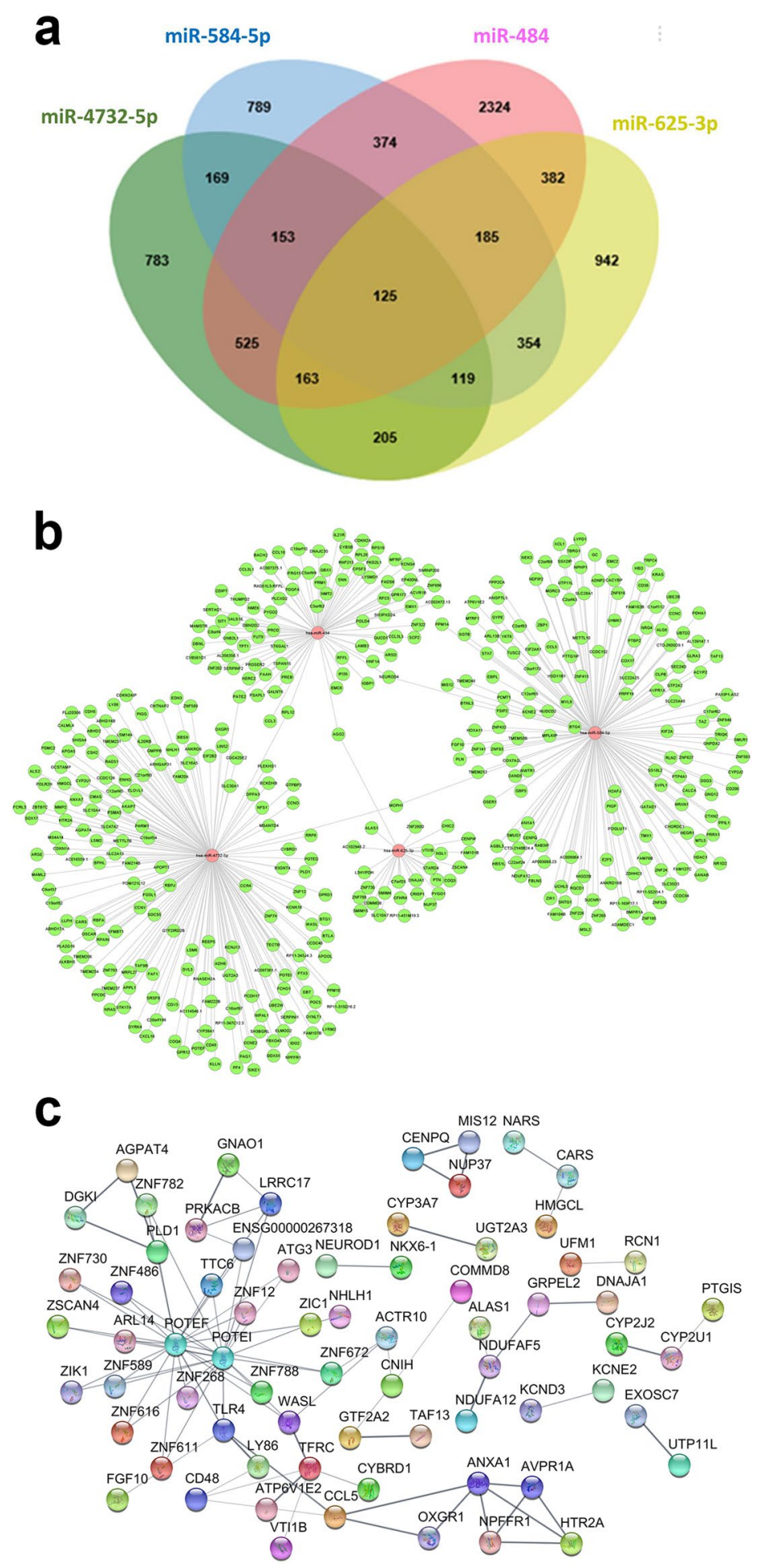

Figure 5. The miRNA-gene network for miRNAs associated with genes related to NTM-PD. (a) Venn diagram showing overlap of gene targets of hsa-miR-484, hsa-miR-584-5p, hsa-miR-625-3p, and hsa-miR-4732-5p. 125 genes were screened to be targeted by the four miRNAs. (b) miRNA-gene network for hsa-miR-484, hsa-miR584-5p, hsa-miR-625-3p, and hsa-miR-4732-5p. The network was built using 125 targeted genes and predicted interactions from the TargetScan databases. Red represents miRNAs, and green represents genes; their relationship is represented by the edges. (c) Protein-protein interaction analysis performed using the Search Tool for the Retrieval of Interacting Genes database revealed that the target genes of hsa-miR-484, hsa-miR584-5p, hsa-miR-625-3p, and hsa-miR-4732-5p may interact with each other. The disconnected nodules are hidden. Bold lines indicate stronger association. 


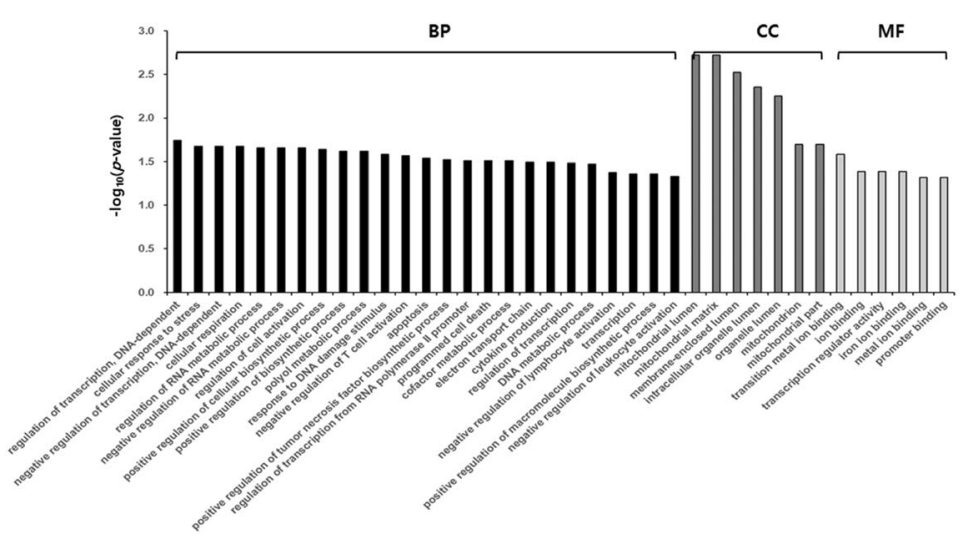

b

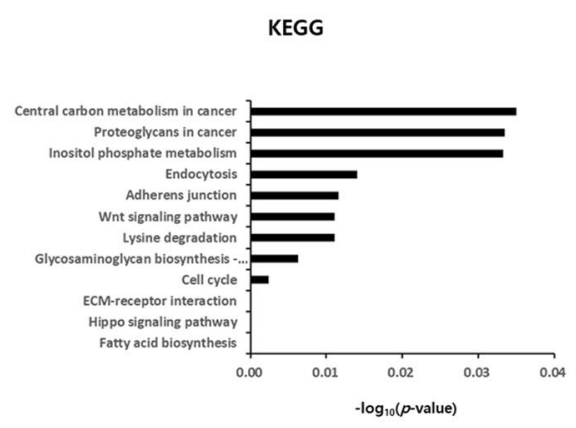

Figure 6. GO enrichment and KEGG pathway analysis for the target genes of selected miRNAs in NTM-PD. (a) GO analysis of hsa-miR-484, hsa-miR-584-5p, hsa-miR-625-3p, and hsa-miR-4732-5p target genes; the $\mathrm{x}$-axis indicates - $\log _{10}$ (p-value), and the $\mathrm{y}$-axis indicates $\mathrm{GO}$ terms; GO, gene ontology; $\mathrm{BP}$, biological processes (black); CC, cellular component (dark gray); MF, molecular function (light gray). (b) KEGG pathway analysis of hsa-miR-484, hsa-miR-584-5p, hsa-miR-625-3p, and hsa-miR-4732-5p target genes; the $\mathrm{x}$-axis indicates $-\log _{10}(\mathrm{p}$-value), and the $\mathrm{y}$-axis indicates KEGG pathway categories.

\section{Materials and Methods}

Study population. Patients with newly diagnosed NTM-PD and who had sera stored at the time of diagnosis of NTM-PD were screened from the NTM Registry of Samsung Medical Center, a 1,979-bed referral hospital in Seoul, South Korea (ClinicalTrials.gov identifier: NCT00970801). To identify NTM-PD-specific miRNAs, we excluded patients with diseases that may influence miRNA expression such as chronic medical disease or malignancy ${ }^{40}$. Finally, 52 patients with NTM-PD (13 each with M. avium, M. intracellulare, M. abscessus, and $M$. massiliense infection) were selected and met the diagnostic criteria for NTM-PD ${ }^{41}$. As healthy controls, 43 hospital employees with no comorbidities were selected. Sera from the study subjects were separated by centrifugation and stored at $-80^{\circ} \mathrm{C}$ for subsequent analysis. Patient recruitment and informed consent were carried out in accordance with approved guidelines from the Institutional Review Board of Samsung Medical Center (IRB No. SMC-2008-09-016).

This study was performed in several phases to identify serum miRNAs that may be useful as markers of NTM-PD (Supplementary Fig. S3). Initially, in the discovery phase, screening was conducted by Illumina small RNA sequencing to select miRNAs with expression patterns that differed between patients with NTM-PD and control subjects. In this step, 15 serum samples were tested to obtain miRNA profiles, with the sera including three samples from patients with NTM for each of the four different pathogens, for a total of 12 patient samples, as well as three samples from control subjects. A panel of miRNAs showing differential expression in patients with NTM-PD and common to PD caused by all four pathogens was subjected to further testing by quantitative reverse-transcription (RT)-polymerase chain reaction (PCR) analysis using sera from additional patients and control subjects.

RNA extraction. Total RNA was isolated from sera using a miRNeasy Mini Kit (Qiagen, Hilden, Germany) following the manufacturer's protocol. Each sample was eluted in RNase-free water. RNA quantity and purity were measured using a NanoDrop ${ }^{\mathrm{TM}}$ ND-2000 (Thermo Fisher Scientific, Waltham, MA, USA), and RNA integrity was assessed using an Agilent Bioanalyzer 2100 (Agilent Technologies, Santa Clara, CA, USA). The RNA samples were stored at $-80^{\circ} \mathrm{C}$ until further analysis.

Illumina high-throughput sequencing. Small RNA libraries were constructed using the NEXTFlex Small RNA Library Prep Kit (Illumina, San Diego, CA, USA) according to the manufacturer's instructions. Briefly, an RNA sequencing library was prepared by cDNA amplification, end-repair, adenylylation of $3^{\prime}$ ends, adapter ligation, and amplification. The libraries were sequenced using the HiSeq 2500 system (Illumina) as 50-base pair single reads.

Small RNA sequencing data analysis. The read data were assessed and pre-processed using FastQC (http://www.bioinformatics.babraham.ac.uk/projects/fastqc), and low-quality bases were trimmed from the $3^{\prime}$ end. Adapter sequences were trimmed and reads shorter than 17 nucleotides were discarded. Trimmed reads were mapped to the reference genome $(\mathrm{GRCH} 38 / \mathrm{hg} 19)$ using Bowtie ${ }^{42}$. The distribution of mapped reads was analyzed by crosschecking with miRBase (http://www.mirbase.org/), Ensembl annotations, and the Rfam database (http://rfam.xfam.org/). Finally, the miRDeep2 program ${ }^{43}$ (https://www.mdc-berlin.de/content/mirdeep2-documentation) was used to identify known miRNAs (based on miRNA data from miRBase v20) to predict novel miRNAs and summarize the read counts for each miRNA in every sample. Differentially expressed miRNAs 
were identified with a threshold fold-change greater than two and adjusted $p$-value $<0.05$. $p$-values were calculated with the DESeq algorithm in the R package for experiments with biological replicates ${ }^{44}$.

Quantification of miRNAs by qRT-PCR. Reverse transcription reactions were performed using a Taq-Man miRNA Reverse Transcription kit (Applied Biosystems, Foster City, CA, USA) and miRNA-specific stem-loop primers in accordance with the manufacturer's instructions. Each reaction mixture for qRT-PCR contained $2.5 \mu \mathrm{L}$ of $2 \times$ TaqMan Universal PCR Master Mix without AmpErase UNG, $0.25 \mu \mathrm{L}$ miRNA-specific primer/probe mix, and $2.25 \mu \mathrm{L}$ diluted RT product (1:15) in a total volume of $5 \mu \mathrm{L}$. Reactions were carried out using the following thermal cycling parameters: $95^{\circ} \mathrm{C}$ for $10 \mathrm{~min}$, followed by 40 cycles of $95^{\circ} \mathrm{C}$ for $15 \mathrm{~s}$, and $60^{\circ} \mathrm{C}$ for $1 \mathrm{~min}$, followed by holding at $4^{\circ} \mathrm{C}$. Raw data were analyzed using SDS Relative Quantification Software version 2.2.3 (Applied Biosystems), generally using the automatic Ct setting for assigning baseline and threshold values for Ct determination. The expression level of each target miRNA was normalized to that of miR-16 (internal control).

miRNA-gene network construction. TargetScan (http://genes.mit.edu/targetscan/index.html) was used to predict genes targeted by the obtained miRNAs. Target genes associated with NTM-PD were screened using the NCBI database (http://www.ncbi.nlm.nih.gov/pubmed/) and Cytoscape (Cytoscape Software, Version 2.8.2, Seattle, WA, USA). The miRNA gene network was constructed using Cytoscape to analyze miRNA-mRNA interactions.

GO analysis and KEGG pathway analysis. GO enrichment was performed for analysis of the biological process, cellular component, and molecular function of target genes based on the GO database (http://www. geneontology.org/). KEGG pathway analysis was performed to identify the enriched pathways of target genes based on the KEGG database (http://www.genome.jp/kegg/). In addition, KEGG pathway annotation of target genes was found using data from the Database for Annotation, Visualization and Integrated Discovery (DAVID) (http://david.abcc.ncifcrf.gov/).

Statistical analysis. Data are presented as the number (\%) for categorical variables and median (interquartile range) for continuous variables. The data were statistically analyzed using SPSS software, version 17.0 (SPSS, Inc., Chicago, IL, USA) or the programs built into the software. Briefly, two-tailed unpaired $t$-tests were performed for one between-group comparison, and one-way analysis of variance was performed for multiple-group comparisons. Receiver operating characteristic (ROC) curve analysis was performed to evaluate the discriminative factor of the miRNAs. The AUC with $95 \%$ confidence interval (CI) were calculated to determine the specificity and sensitivity of discriminative markers of NTM-PD. ROC curve and logistic regression model were calculated by using the MedCalc Software (Version 19.1.13, Belgium). A default $p<0.05$ was considered statistically significant.

\section{Data availability}

All data generated or analysed during this study are included in this published article and its Supplementary Information Files.

Received: 26 July 2019; Accepted: 7 February 2020;

Published online: 21 February 2020

\section{References}

1. Adjemian, J., Daniel-Wayman, S., Ricotta, E. \& Prevots, D. R. Epidemiology of Nontuberculous Mycobacteriosis. Semin. Respir. Crit. Care Med. 39, 325-335 (2018).

2. Prevots, D. R. \& Marras, T. K. Epidemiology of human pulmonary infection with nontuberculous mycobacteria: a review. Clin Chest Med 36, 13-34 (2015).

3. Henkle, E. et al. Patient-centered research priorities for pulmonary nontuberculous mycobacteria (NTM) infection. An NTM research consortium workshop report. Ann Am Thorac Soc 13, S379-384 (2016)

4. Daniel-Wayman, S. et al. Advancing Translational Science for Pulmonary Nontuberculous Mycobacterial Infections. A Road Map for Research. Am. J. Respir. Crit. Care Med. 199, 947-951 (2019).

5. O’Connell, R. M., Rao, D. S., Chaudhuri, A. A. \& Baltimore, D. Physiological and pathological roles for microRNAs in the immune system. Nat Rev Immunol 10, 111-122 (2010).

6. Watanabe, Y. \& Kanai, A. Systems biology reveals microRNA-mediated gene regulation. Front Genet 2, 29 (2011).

7. Hrustincova, A., Votavova, H. \& Dostalova Merkerova, M. Circulating microRNAs: methodological aspects in detection of these biomarkers. Folia Biol (Praha) 61, 203-218 (2015).

8. Verma, P., Pandey, R. K., Prajapati, P. \& Prajapati, V. K. Circulating microRNAs: potential and emerging biomarkers for diagnosis of human infectious diseases. Front Microbiol 7, 1274 (2016).

9. Belver, L., Papavasiliou, F. N. \& Ramiro, A. R. MicroRNA control of lymphocyte differentiation and function. Curr Opin Immunol 23, 368-373 (2011).

10. Singh, Y. et al. Mycobacterium tuberculosis controls microRNA-99b (miR-99b) expression in infected murine dendritic cells to modulate host immunity. J Biol Chem 288, 5056-5061 (2013).

11. Gilad, S. et al. Serum microRNAs are promising novel biomarkers. PLoS One 3, e3148 (2008).

12. Mitchell, P. S. et al. Circulating microRNAs as stable blood-based markers for cancer detection. Proc Natl Acad Sci USA 105, 10513-10518 (2008).

13. Qi, Y. et al. Altered serum microRNAs as biomarkers for the early diagnosis of pulmonary tuberculosis infection. BMC Infect Dis $\mathbf{1 2}$, 384 (2012).

14. Miotto, P. et al. miRNA signatures in sera of patients with active pulmonary tuberculosis. PLoS One 8, e80149 (2013).

15. Fu, Y., Yi, Z., Wu, X., Li, J. \& Xu, F. Circulating microRNAs in patients with active pulmonary tuberculosis. J Clin Microbiol 49, 4246-4251 (2011).

16. Wu, J. et al. Analysis of microRNA expression profiling identifies miR-155 and miR-155* as potential diagnostic markers for active tuberculosis: a preliminary study. Hum. Immunol. 73, 31-37 (2012). 
17. Zhang, H. et al. Identification of serum microRNA biomarkers for tuberculosis using RNA-seq. PLoS One 9, e88909 (2014).

18. Lyu, L. et al. Small RNA Profiles of Serum Exosomes Derived From Individuals With Latent and Active Tuberculosis. Front Microbiol 10, 1174 (2019).

19. Sabir, N. et al. MiRNAs in Tuberculosis: new avenues for diagnosis and host-directed therapy. Front Microbiol 9, 602 (2018).

20. Alipoor, S. D. et al. Bovis bacillus calmette-guerin (BCG) infection induces exosomal miRNA release by human macrophages. $J$ Transl Med 15, 105 (2017).

21. Wu, L. S. et al. Systematic expression profiling analysis identifies specific microRNA-gene interactions that may differentiate between active and latent tuberculosis infection. Biomed Res Int 2014, 895179 (2014).

22. Latorre, I. et al. A novel whole-blood miRNA signature for a rapid diagnosis of pulmonary tuberculosis. Eur Respir J45, 1173-1176 (2015).

23. Zhou, M. et al. Circulating microRNAs as biomarkers for the early diagnosis of childhood tuberculosis infection. Mol Med Rep 13, 4620-4626 (2016).

24. Lv, Y. et al. Sputum and serum microRNA-144 levels in patients with tuberculosis before and after treatment. Int. J. Infect. Dis. 43, 68-73 (2016)

25. Wagh, V., Urhekar, A. \& Modi, D. Levels of microRNA miR-16 and miR-155 are altered in serum of patients with tuberculosis and associate with responses to therapy. Tuberculosis (Edinb) 102, 24-30 (2017).

26. Sharbati, J. et al. Integrated microRNA-mRNA-analysis of human monocyte derived macrophages upon Mycobacterium avium subsp. hominissuis infection. PLoS One 6, e20258 (2011).

27. Nishimura, T. et al. Hsa-miR-346 is a potential serum biomarker of Mycobacterium avium complex pulmonary disease activity. $J$ Infect Chemother 23, 703-708 (2017).

28. Kang, S. M., Choi, J. W., Lee, Y., Hong, S. H. \& Lee, H. J. Identification of microRNA-size, small RNAs in Escherichia coli. Curr. Microbiol. 67, 609-613 (2013).

29. Lagos-Quintana, M., Rauhut, R., Lendeckel, W. \& Tuschl, T. Identification of novel genes coding for small expressed RNAs. Science 294, 853-858 (2001).

30. Wang, K. et al. MiR-484 regulates mitochondrial network through targeting Fis1. Nat Commun 3, 781 (2012)

31. Li, T., Ding, Z. L., Zheng, Y. L. \& Wang, W. MiR-484 promotes non-small-cell lung cancer (NSCLC) progression through inhibiting Apaf-1 associated with the suppression of apoptosis. Biomed Pharmacother 96, 153-164 (2017).

32. Fils-Aime, N. et al. MicroRNA-584 and the protein phosphatase and actin regulator 1 (PHACTR1), a new signaling route through which transforming growth factor-beta Mediates the migration and actin dynamics of breast cancer cells. J. Biol. Chem. 288, 11807-11823 (2013).

33. Yang, L., Pang, Y. \& Moses, H. L. TGF-beta and immune cells: an important regulatory axis in the tumor microenvironment and progression. Trends Immunol 31, 220-227 (2010).

34. Roth, C. et al. Low levels of cell-free circulating miR-361-3p and miR-625* as blood-based markers for discriminating malignant from benign lung tumors. PLoS One 7, e38248 (2012).

35. Wang, J. et al. Identification of potential urine proteins and microRNA biomarkers for the diagnosis of pulmonary tuberculosis patients. Emerg Microbes Infect 7, 63 (2018).

36. Zhang, K., Wang, Y. W. \& Ma, R. Bioinformatics analysis of dysregulated microRNAs in the nipple discharge of patients with breast cancer. Oncol Lett 13, 3100-3108 (2017).

37. Ranjbar, S., Jasenosky, L. D., Chow, N. \& Goldfeld, A. E. Regulation of Mycobacterium tuberculosis-dependent HIV-1 transcription reveals a new role for NFAT5 in the toll-like receptor pathway. PLoS Pathog 8, e1002620 (2012).

38. Rose-John, S., Winthrop, K. \& Calabrese, L. The role of IL-6 in host defence against infections: immunobiology and clinical implications. Nat Rev Rheumatol 13, 399-409 (2017).

39. Zhang, Z. et al. Variable selection in Logistic regression model with genetic algorithm. Ann Transl Med 6, 45 (2018).

40. Shrestha, S. et al. A systematic review of microRNA expression profiling studies in human gastric cancer. Cancer Med 3, 878-888 (2014).

41. Griffith, D. E. et al. An official ATS/IDSA statement: diagnosis, treatment, and prevention of nontuberculous mycobacterial diseases. Am. J. Respir. Crit. Care Med. 175, 367-416 (2007).

42. Langmead, B. Aligning short sequencing reads with Bowtie. Curr Protoc Bioinformatics Chapter 11, Unit 1117 (2010).

43. Friedlander, M. R., Mackowiak, S. D., Li, N., Chen, W. \& Rajewsky, N. miRDeep2 accurately identifies known and hundreds of novel microRNA genes in seven animal clades. Nucleic Acids Res. 40, 37-52 (2012).

44. Anders, S. \& Huber, W. Differential expression analysis for sequence count data. Genome Biol. 11, R106 (2010).

\section{Acknowledgements}

This work was supported by the National Research Foundation of Korea (NRF) funded by the Korean government (MSIT) (NRF-2018R1A2A1A05018309) and the Ministry of Education (NRF-2016R1A6A3A11932695).

\section{Author contributions}

S.A.H., S.J.S. and W.-J.K. designed and performed experiment, analyzed data, and wrote the manuscript. B.W.J., S.-Y.K., S.M.M., B.Y., O.J.K. and C.L.D. contributed to the discussion and interpretation of the results.

\section{Competing interests}

Dr. Charles L. Daley has received grants from Insmed, Inc., and served on Advisory Boards for Insmed, Inc., Johnson and Johnson, Spero, and Horizon, not associated with the submitted work. Dr. Won-Jung Koh has received a consultation fee from Insmed, Inc., for the Insmed Advisory Board Meeting, not associated with the submitted work. Otherwise, we have no conflicts of interest to declare.

\section{Additional information}

Supplementary information is available for this paper at https://doi.org/10.1038/s41598-020-60132-0.

Correspondence and requests for materials should be addressed to S.J.S. or W.-J.K.

Reprints and permissions information is available at www.nature.com/reprints.

Publisher's note Springer Nature remains neutral with regard to jurisdictional claims in published maps and institutional affiliations. 
(c) (i) Open Access This article is licensed under a Creative Commons Attribution 4.0 International License, which permits use, sharing, adaptation, distribution and reproduction in any medium or format, as long as you give appropriate credit to the original author(s) and the source, provide a link to the Creative Commons license, and indicate if changes were made. The images or other third party material in this article are included in the article's Creative Commons license, unless indicated otherwise in a credit line to the material. If material is not included in the article's Creative Commons license and your intended use is not permitted by statutory regulation or exceeds the permitted use, you will need to obtain permission directly from the copyright holder. To view a copy of this license, visit http://creativecommons.org/licenses/by/4.0/.

(C) The Author(s) 2020 